Proc. of 12th International Workshop on Positron and Positronium Chemistry, August 28-September 1, 2017, Lublin, Poland

\title{
Positronium Probing of Pores in Zirconia Nanopowders
}

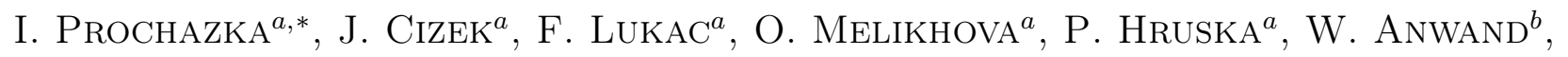 \\ M.O. LiedKe ${ }^{b}$, T.E. KonstantinOva ${ }^{c}$ And I.A. DANilenkO ${ }^{c}$ \\ ${ }^{a}$ Charles University, Faculty of Mathematics and Physics, Department of Low Temperature Physics, \\ V Holesovickach 2, CZ-180 00 Praha 8, Czech Republic \\ ${ }^{b}$ Helmholtz-Zentrum Dresden-Rossendorf, Institute of Radiation Physics, Bautzner Landstr. 400, 01328 Dresden, \\ Germany
}

${ }^{c}$ National Academy of Sciences of Ukraine, Donetsk Institute for Physics and Engineering named after O.O. Galkin, Nauki av. 46, 03028 Kiev, Ukraine

\begin{abstract}
In the present paper, conventional positron lifetime measurements on selected zirconia-based nanopowders are reported. The nanopowders were doped with various metal cations $\left(\mathrm{Y}^{3+}, \mathrm{Eu}^{3+}, \mathrm{Gd}^{3+}, \mathrm{Lu}^{3+}\right.$ and $\left.\mathrm{Mg}^{2+}\right)$. Lifetime experiments were conducted in air and supplemented with mass density measurements. In a range of lifetimes, from a few ns to $\approx 70 \mathrm{~ns}$, up to two individual lifetime components could be identified. Such observations confirmed positronium (Ps) formation with subsequent ortho-Ps pick-off annihilation as well as the occurrence of pores of different size. Pore sizes were estimated using a shape-free model of the correlation between pore size and ortho-Ps lifetime. The origins of pores are discussed on the basis of the ortho-Ps data in combination with the results of mass density measurements.
\end{abstract}

DOI: 10.12693/APhysPolA.132.1564

PACS/topics: 81.07.Wx, 61.46.Hk, 78.70.Bj

\section{Introduction}

Metal-oxide (MO) nanopowders serve as essential substances for preparation of many functional materials and devices. In this regard, it is enough to mention materials used in ceramic industry, or components for electronics and optics. To a great extent, required as well as undesired properties of MOs materials are influenced by defects in the material of a submicrometer size. Some of these defects are inherent to the crystal lattice: monovacancies, vacancy clusters and complexes, dislocations, interstitials. In nanopowders, grain boundaries (GBs) occupy large volume fraction of material. In this case, vacancy-like defects associated with GBs, voids of a fewvacancy cluster size at junctions of three or more GBs (triple points) and large interparticle open spaces (nanoor mesopores) play an increased role. It is apparent that thorough characterisation of submicrometer-sized defects and other microstructure aspects is a prerequisite of successful implementation of MOs-based nanomaterials in practical applications. The diversity of open-volume structures that may be encountered in MO nanopowders makes positron annihilation spectroscopy techniques irreplaceable in microstructure investigations into these materials.

Among MO substances, zirconium dioxide $\left(\mathrm{ZrO}_{2}\right.$, zirconia) has a wide range of applications $[1,2]$ due to its advantageous thermal, electrical and mechanical features: a wide energy gap, a high melting point, low thermal and

*corresponding author; e-mail: ivan.prochazka@mff.cuni.c electric conductivity, appreciable hardness at reasonable toughness. The functional properties of zirconia-based nanomaterials can be improved by proper doping with other MO admixtures as well as by appropriate thermal treatments of the initial nanopowders (calcination, annealing, sintering). Microstructure changes caused by such variations in material processing are the subject of detailed investigations

The present study aims to perform detailed measurements and analysis of positron lifetime (PLT) spectra on selected pressure-compacted nanopowders containing $\mathrm{ZrO}_{2}$ as the base compound doped with other MOs $\left(\mathrm{Y}_{2} \mathrm{O}_{3}, \mathrm{Eu}_{2} \mathrm{O}_{3}, \mathrm{Gd}_{2} \mathrm{O}_{3}, \mathrm{Lu}_{2} \mathrm{O}_{3}, \mathrm{MgO}\right)$ with particular emphasis on the long lifetime components corresponding to positronium (Ps) pick-off annihilation. The PLT experiments were supplemented by mass density (MD) measurements. Some preliminary PLT results concerning Ps formation and the pick-off process were mentioned in our previous papers [3-5].

\section{Experiments}

\subsection{Samples}

The initial nanopowders were prepared by the coprecipitation method, described in Ref. [6]. The nanopowders were calcined in air at temperature $T_{c}\left[{ }^{\circ} \mathrm{C}\right]$ for time $\tau[\mathrm{h}]$. The average size of nanoparticles (diameter $d[\mathrm{~nm}]$ ) was estimated $[3,4]$ from transmission electron microscopy or X-ray diffraction. The calcined nanopowders were compacted under different uniaxial pressures $\mathrm{P}$ (2.5 to $10 \mathrm{kbar}$ ) to form disk-shaped tablets of $\approx 15 \mathrm{~mm}$ in diameter and $\approx 2 \mathrm{~mm}$ in thickness. Basic characteristics of the nanopowders included in the present work are summarized in Table I. 
TABLE I

Basic characteristics of nanopowders investigated in the present work.

\begin{tabular}{|c|c|c|c|}
\hline Composition; symbol, $\left(\right.$ phase $\left.^{a}\right)$ & $P$ & $d$ & $T_{c} / \tau$ \\
\hline $\mathrm{ZrO}_{2} ; m-\mathrm{ZrO}_{2},(\mathrm{M})$ & 2.5 & 15 & \\
\hline$\overline{\mathrm{ZrO}_{2}+3 \mathrm{~mol} \% \mathrm{Y}_{2} \mathrm{O}_{3} ; t-\mathrm{YSZ},(\mathrm{T})}$ & $\begin{array}{c}5 \\
10 \\
\end{array}$ & 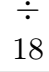 & $700 / 2$ \\
\hline $\mathrm{ZrO}_{2}+10 \mathrm{~mol} \% \mathrm{MgO} ; \mathrm{MgSZ}(98 \mathrm{M} 2 \mathrm{~T})$ & 5 & 10 & $500 / 2$ \\
\hline $\begin{array}{lc} & \text { EuSZ }(98.5 \mathrm{~T} 1.5 \mathrm{M}) \\
\mathrm{ZrO}_{2}+3 \mathrm{~mol} \% \mathrm{RE}_{2} \mathrm{O}_{3} ; & \operatorname{GdSZ}(95 \mathrm{~T} 5 \mathrm{M}) \\
& \operatorname{LuSZ}(87 \mathrm{~T} 13 \mathrm{M})\end{array}$ & 2 & 18 & $700 / 2$ \\
\hline
\end{tabular}

\subsection{Measurements and data evaluation}

Positron sources were manufactured from a carrier-free ${ }^{22} \mathrm{NaCl}$ solution purchased from iThemba LABS (South Africa). A drop of the solution containing $\approx 1 \mathrm{MBq}$ of ${ }^{22} \mathrm{Na}$ was dried and sealed between two Kapton ${ }^{\circledR}$ foils of $7.4 \mu \mathrm{m}$ thickness. The diameter of the activity spot did not exceed $\approx 2 \mathrm{~mm}$.

PLT spectra were accumulated using a delayedcoincidence spectrometer similar to that described in Ref. [7]. The detector part of the spectrometer utilised $\mathrm{BaF}_{2}$ scintillators coupled to photomultiplier tubes. PLT measurements were conducted in normal-pressure air at room temperature. More than $5 \times 10^{6}$ coincidence events were collected in each PLT spectrum.

The dominating part of PLT spectra observed in many nanopowdered metal oxides (including zirconia nanopowders) originates from annihilations of positrons that have not formed Ps. It exhausts typically more than $80 \%$ of spectral intensity which is distributed into two components characterised with lifetimes of $\approx 0.2$ and $\approx 0.4$ ns. These components arise, respectively, from annihilations of positrons localised in vacancy-like defects situated along grain boundaries and somewhat larger open spaces occurring at intersections of three grain boundaries $[3,4]$. Both these components should vanish for positron ages $t$ above $\approx 7 \mathrm{~ns}$. When Ps formation takes place in the studied material, the pick-off annihilations of ortho-Ps $(o \mathrm{Ps})$ make an additional contribution to the PLT spectrum. The $o$ Ps part of the spectrum is composed typically of several components with lifetimes above 1 ns which may frequently reach values of $\approx 100 \mathrm{~ns}$. Such long-lived components represent virtually the sole contribution in the $t>7 \mathrm{~ns}$ region of the spectrum. In order that full $o$ Ps spectrum could be recorded, a time window width of $\approx 1 \mu \mathrm{s}$ (8K channels of $0.124 \mathrm{~ns}$ channel width each) was chosen in the PLT measurements of the present work. In this mode, however, the spectrometer time resolution is deteriorated to $\approx 0.45 \mathrm{~ns}$ (FWHM). Information about the lifetime components below $0.5 \mathrm{~ns}$, obtainable under these circumstances (channel width, time resolution), becomes thus much poorer in comparison with measurements made in a more favourable spectrometer setup for the short lifetimes. Since the $o$ Ps lifetime components only are addressed in the present work, PLT spectra anal- ysis was restricted to positron age region of $t>7 \mathrm{~ns}$ where short-lived components $(<0.5 \mathrm{~ns})$ as well as time resolution function play no important role. A maximum likelihood method, similar to that of Ref. [8], but involving only the lifetimes and relative intensities of the $o$ Ps components as fitted parameters, was used to decompose the $o$ Ps part of the PLT spectrum. In the calculations of the $o$ Ps intensities, the positron source contribution (kapton foils plus ${ }^{22} \mathrm{Na}$ salt) was subtracted. The source contribution was determined from measurements on a well-annealed $\alpha$-iron reference sample and recalculated as $11 \pm 1 \%$ to the zirconia specimens using the method suggested in [9]. The resulted lifetimes and relative intensities of the $o$ Ps components were summarized in Table II. The errors quoted in the table correspond to model fitting errors. Systematic errors originated from potential model inaccuracy were estimated to be less than $5 \%$ for the shorter $o$ Ps component and negligible for the longest one. An example of a PLT spectrum measured in the present work is given in Fig. 1.

TABLE II

Summary of mass density and oPs lifetime measurements carried out in the present work. Sample abbreviations were introduced in Table 1. Meaning of symbols in the Table header was explained in text. Experimental errors (1 standard deviation) are shown in units of the last significant digit.

\begin{tabular}{c|c|c|c|c|c|c|c|c}
\hline \hline Sample & $\begin{array}{c}P \\
{[\mathrm{kbar}]}\end{array}$ & $\begin{array}{c}\rho \\
{\left[\mathrm{g} / \mathrm{cm}^{3}\right]}\end{array}$ & $\begin{array}{c}\tau_{o \mathrm{Ps}, 1} \\
{[\mathrm{~ns}]}\end{array}$ & $\begin{array}{c}I_{o \mathrm{Ps}, 1} \\
{[\%]}\end{array}$ & $\begin{array}{c}R_{1} \\
{[\mathrm{~nm}]}\end{array}$ & $\begin{array}{c}\tau_{o \mathrm{Ps}, 2} \\
{[\mathrm{~ns}]}\end{array}$ & $\begin{array}{c}I_{o \mathrm{Ps}, 2} \\
{[\%]}\end{array}$ & $\begin{array}{c}R_{2} \\
{[\mathrm{~nm}]}\end{array}$ \\
\hline \multirow{3}{*}{$m$-ZrO $_{2}$} & 2.5 & $2.759(7)$ & $7.6(6)$ & $1.0(2)$ & $0.61(3)$ & $75.4(15)$ & $7.7(2)$ & $8.7(11)$ \\
& 5.0 & $3.01(3)$ & $7.2(9)$ & $0.8(2)$ & $0.59(4)$ & $72.7(20)$ & $7.9(2)$ & $7.0(10)$ \\
& 10.0 & $3.302(6)$ & $6.7(6)$ & $1.0(2)$ & $0.57(3)$ & $69.2(14)$ & $7.4(2)$ & $5.6(5)$ \\
\hline \multirow{3}{*}{$t$-YSZ } & 2.5 & $2.556(8)$ & $7.6(8)$ & $0.7(1)$ & $0.61(3)$ & $74.2(13)$ & $8.5(1)$ & $7.9(8)$ \\
& 5.0 & $2.922(5)$ & $7.8(11)$ & $0.7(2)$ & $0.61(4)$ & $70.7(22)$ & $7.7(2)$ & $6.1(9)$ \\
& 10.0 & $3.347(6)$ & $6.5(15)$ & $0.4(2)$ & $0.56(7)$ & $65.3(11)$ & $8.1(2)$ & $4.5(3)$ \\
\hline EuSZ & 5.0 & $3.05(1)$ & $9.2(18)$ & $0.6(2)$ & $0.66(6)$ & $65.3(27)$ & $7.3(2)$ & $4.5(6)$ \\
\hline GdSZ & 5.0 & $2.866(8)$ & $8.1(10)$ & $0.6(2)$ & $0.62(4)$ & $73.3(13)$ & $7.9(2)$ & $7.3(7)$ \\
\hline LuSZ & 5.0 & $3.10(1)$ & $7.8(16)$ & $0.4(2)$ & $0.61(6)$ & $75.3(22)$ & $6.0(2)$ & $8.6(16)$ \\
\hline MgSZ & 5.0 & $2.25(15)$ & $5.6(6)$ & $0.7(1)$ & $0.52(3)$ & $64.9(14)$ & $6.7(10)$ & $4.4(3)$
\end{tabular}

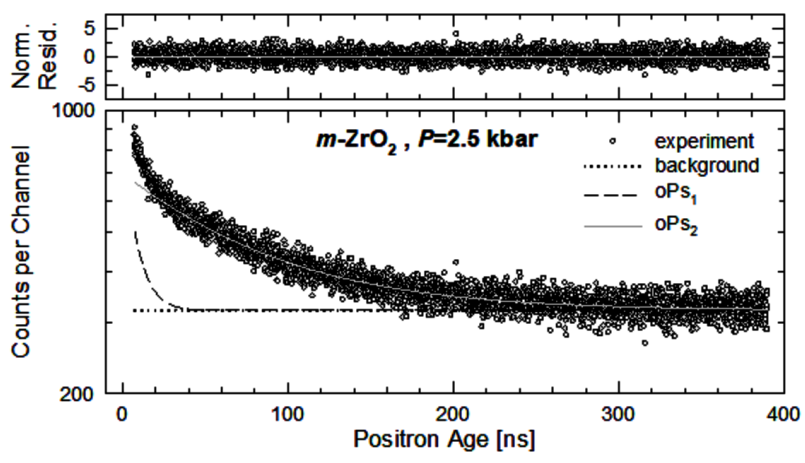

Fig. 1. Lower panel: An example of PLT spectrum (circles) measured in the present work $\left(m-\mathrm{ZrO}_{2}, P=\right.$ $2.5 \mathrm{kbar}, t>7.5 \mathrm{~ns}$ ) and the two fitted $o$ Ps components (lines, superimposed on fitted constant background). Upper panel: Residuals between experiment and fitted model, normalised to root mean square deviations of data points. 
Mass densities were determined from the precise weighting and size measurements of pellets and these were compared with previously published theoretical mass densities and the mass density measured for a reference $t$-YSZ single crystal of similar chemical composition.

\section{Results and discussion}

The measured mass densities, $\rho$, of pressure-compacted nanopowders used in the present study are shown in Table II (coll. 3). One can see from the table that the present experimentally measured mass densities have a range between 2.5 and $3.3 \mathrm{~g} / \mathrm{cm}^{3}$ with a mean value of $\approx 2.9 \mathrm{~g} / \mathrm{cm}^{3}$. The theoretical densities published so far for the tetragonal and cubic $\mathrm{ZrO}_{2}$ lattices [10, 11] appear to be close to each other, $\rho_{t h}=6.10$ and $6.09 \mathrm{~g} / \mathrm{cm}^{3}$ respectively, while a somewhat lower value of $\rho_{t h}=5.85 \mathrm{~g} / \mathrm{cm}^{3}$ has been reported for the monoclinic $\mathrm{ZrO}_{2}$ lattice [11]. In addition, the mass density of the reference $t$-YSZ single crystal was measured as $5.98 \pm 0.01 \mathrm{~g} / \mathrm{cm}^{3}$ in the present work which is close to the theoretical value. Comparison of the measured and theoretical mass densities of the compacted nanopowders (Table II) show that the nanopowders have a mass density of about $50 \%$ of the theoretical limits as well as the reference single-crystal value. The measured mass densities are far below a value of $74 \%$ which would be expected for a system of ideally packed equal-sized hardsphere particles, implied by solutions of the Kepler conjecture problem [12]. Models of random packing of uniform hard spheres have also been studied both theoretically as well as experimentally and maximum densities of $\approx 55$ and $\approx 63-64 \%$ of the theoretical density value are typically encountered in cases of so called random loose packing (RLP) and random close packing (RCP), respectively [13]. The experimental mass densities shown in Table II seem to be in a reasonable consistency with a value of $\approx 2.8 \mathrm{~g} / \mathrm{cm}^{3}$ expected for a nanopowder composed of RCP packed aggregates of ideally packed initial nanoparticles. The net RLP mechanism with no aggregation of initial nanoparticles leads to an expected density of $\approx 3.2 \mathrm{~g} / \mathrm{cm}^{3}$ which seems less consistent with experimental data, but cannot be fully ruled out, though.

Positron lifetimes greater than $1 \mathrm{~ns}$ were observed in pressure-compacted nanopowders studied in the present work. Thus, Ps formation in these nanopowders and oPs decay via the pick-off annihilation process are clearly observed in the present PLT data. Corresponding o-Ps lifetimes and relative intensities are also listed in Table II (colls. 4, 5 and 7,8 ). It can be seen from the table that two o-Ps lifetime components could be resolved in the PLT spectra for all the nanopowders. The shorter component exhibited o-Ps lifetimes $\tau_{o \mathrm{Ps}, 1} \approx 7.5 \mathrm{~ns}$ and relative intensity $I_{o \mathrm{Ps}, 1} \approx 0.7 \%$, while the longer ones feature lifetime $\tau_{o \mathrm{Ps}, 2} \approx 70 \mathrm{~ns}$ and relative intensity $I_{o \mathrm{Ps}, 2} \approx 7.5 \%$.

Based on the ideas presented in Refs. [14, 15], we suppose that in bulk compacted nanopowders like in many other bulk porous materials, Ps formation takes place near internal surfaces in the material and is followed by a subsequent repulsion of Ps atoms into a neighbour open volume (nano- or mesopore). Inside such a cavity, Ps atoms become thermalized and remain confined there until positron annihilation. A positive correlation between the o-Ps lifetime and cavity size is well-proven and several semi-empirical relations between o-Ps lifetime and cavity size parameters have been proposed to date [1622 . In the present paper, we make use of a simple shapefree model suggested recently by Wada and Hyodo [22] for pore size estimation using experimental $o$ Ps lifetimes. In their model, the primary measure of pore size is the mean free length of the pore, $\bar{L}$, which can be eventually transformed into radius $R$ of a spherical pore as $\bar{L}=4 R / 3$. In what follows, the pore size is expressed in terms of pore radius $R$ estimated from the present oPs lifetime data using the Wada and Hyodo formula [22]. Since the present PLT measurements were conducted in air, a correction of the experimental lifetimes due to the ortho-para quenching of o-Ps was applied. A quenching rate of $\lambda_{q}=0.00492 \pm 0.00040 \mathrm{~ns}^{-1}$ [21] was adopted. This quenching rate appears to be consistent with data revealed in our comparative measurements on $\mathrm{MgSZ}$ nanopowders performed in normal-pressure air and in rough vacuum [5].

The PLT results summarized in Table II indicate that two kinds of pores, differing mutually in size, exist in the nanopowders under study. The estimated pore radii, $R_{i}(i=1,2)$, are given in Table II, colls. 6 and 9. The radii $R_{1}$ of the smaller pores are around $\approx 0.6 \mathrm{~nm}$, while the larger ones, $R_{2}$, fall between $\approx 4.5$ and $\approx 8.5 \mathrm{~nm}$. At least two kinds of pores may be expected in the pressurecompacted nanopowders [23]: (i) small pores of a few nanometers in size occurring among primary nanoparticles and (ii) relatively larger pores among aggregates of several nanoparticles. Thus an occurrence of two kinds of pores, indicated by the present PLT measurements, may again be regarded as pointing to an issue of aggregation of primary particles in compacted nanopowders under study. In such approach, the smaller radii, $R_{1}$, correspond to pores among primary particles while the larger ones, $R_{2}$, are attributed to pores among particle aggregates. However, our knowledge about nanoparticle aggregation is still rather incomplete. A simple model of primary particle aggregation was applied to several compacted nanopowders in Ref. [23]. It seems, however, that the order of packing of particles or their aggregates, which is obviously significantly influenced by the applied compaction pressure, has to be inherent to the model. Otherwise, the model represents an oversimplified picture of a real system. As discussed above, the present mass density data do not seem to support the ideal packing of aggregates. The origin of the $\tau_{o \mathrm{Ps}, 1}$-components is even more unclear, because pore sizes deduced from the present PLT data are significantly lower than those expected in [23] for the smaller cavities within the model. It seems likely that another mechanism should co-control formation of the smaller pores. 


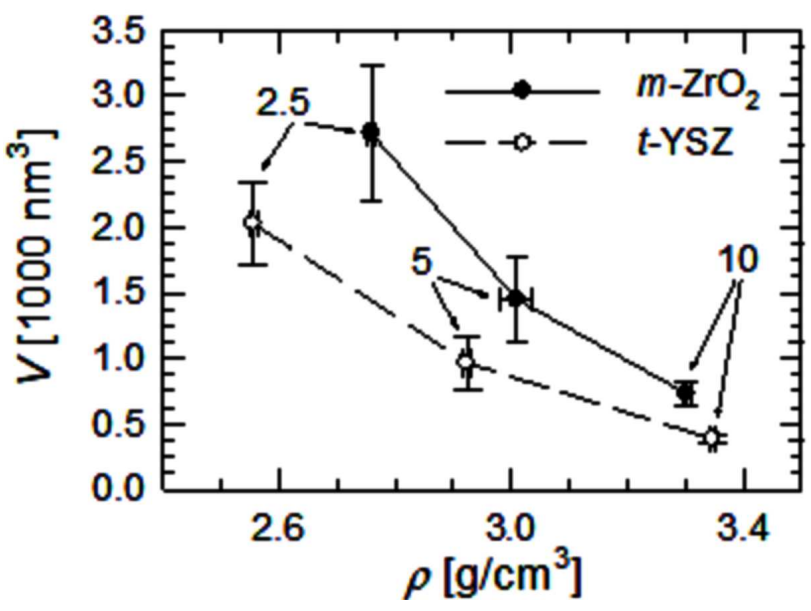

Fig. 2. Correlation between the volume of larger pores, $V$, and mass density, $\rho$, found in the present work for $m-\mathrm{ZrO}_{2}$ and $t$-YSZ nanopowders compacted under different pressure. Numbers inserted denote the applied compaction pressure in kbar.

The measured mass densities of $m-\mathrm{ZrO}_{2}$ and $t$-YSZ nanopowders compacted under different pressure $P$ are plotted against pore volumes calculated from $R_{2}$-values of Table II assuming a spherical pore shape, in Fig. 2. This figure illustrates a densification of both $m-\mathrm{ZrO}_{2}$ and $t$-YSZ nanopowders with increasing compaction pressure which is accompanied by a decrease in pore volume by a factor of about two. Such behaviour seems to reflect a gradual pressure-induced breakdown of aggregates into smaller units which should lead subsequently to a diminishing of inter-aggregate open volumes. At the same time, these results illustrate the importance of a proper choice of compacting pressure for manufacturing nanopowders intended for particular applications

\section{Summary}

In the present paper, the zirconia nanopowders doped with several metal oxides $\left(\mathrm{Y}_{2} \mathrm{O}_{3}, \mathrm{Eu}_{2} \mathrm{O}_{3}, \mathrm{Gd}_{2} \mathrm{O}_{3}, \mathrm{Lu}_{2} \mathrm{O}_{3}\right.$, $\mathrm{MgO})$ and compacted under various uniaxial pressures from 2.5 up to 10 kbar were investigated by means of positron lifetime spectroscopy combined with mass density measurements. Positronium (Ps) formation was seen in all the nanopowders studied and observed o-Ps lifetimes were used to characterise the sizes of nano- and mesopores. The results indicate the occurrence of particle aggregation. Decrease of pore size with increasing compacting pressure also suggest pressure-induced aggregate breakdown in pure zirconia and yttria doped zirconia nanopowders.

\section{Acknowledgments}

This work was financially supported by The Czech Sci. Foundation (project P108/12/G043) and The National Acad. Sci. of Ukraine (project $89 / 12-\mathrm{H}$ ).

\section{References}

[1] Science and Technology of zirconia V, Eds.: S.P. Badwal, M.J. Bannister, R.H.J. Hannink, Technomic Pub. Co., Lancaster (Pennsylvania) 1993.

[2] M. Fernandez-Garcia, J.A. Rodriguez: Metal Oxide Nanoparticles, in: Encyclopedia of Inorganic Chemistry, Wiley, 2009.

[3] J. Cizek, O. Melikhova, I. Prochazka, J. Kuriplach, R. Kuzel, G. Brauer, W. Anwand, T.E. Konstantinova, I.A. Danilenko, Phys. Rev. B 81, 024116 (2010).

[4] I. Prochazka, J. Cizek, O. Melikhova, T.E. Konstantinova, I.A. Danilenko, I.A. Yashchishyn, J. Am. Ceram. Soc. 97, 982 (2014).

[5] I. Prochazka, J. Cizek, O. Melikhova, P. Hruska, W. Anwand, T.E. Konstantinova, I.A. Danilenko, Def. Diff. Forum 373, 295 (2016).

[6] T. Konstantinova, I. Danilenko, V. Glazunova, G. Volkova, O. Gorban, J. Nanopart. Res 13, 4015 (2011).

[7] F. Becvar, J. Cizek, L. Lestak, I. Novotny, I. Prochazka, F. Sebesta, Nucl. Instr. Meth. Phys. Res. A 443, 557 (2000).

[8] I. Prochazka, I. Novotny, F. Becvar, Mater. Sci. Forum 255 -257, 772 (1997).

[9] H. Surbeck, Helv. Phys. Acta 50, 705 (1977).

[10] S. Ran, L. Winnubst, W. Wiratha, D.H.A. Blank, J. Amer. Ceram. Soc. 89, 151 (2006).

[11] RSC, Zinc and Zirconia, Zirconia, p. 27.

[12] T.C. Hales, J. Harrison, S. McLaughlin, T. Nipkow, S. Obua, R. Zumkeller, Discr. Comput. Geometry 44, (2010).

[13] P. Wang, C. Song, Y. Jin, H.A. Makse, Physica A 390, 427 (2011).

[14] A. Dupasquier, in: Positron Solid State Physics, Proc. Internat. School of Physics ÂTEEnrico FermiÂt', C. LXXXIII, Eds.: W. Brandt, A. Dupasquier, North-Holland, Amsterdam 1983, p. 510.

[15] C, Dauwe, G. Consolati, J. Kansy, B. Van Waeyenberge, Physics Letters A 238, 379 (1998).

[16] M. Eldrup, D. Lightbody, J.N. Sherwood, Chem. Phys. 63, 51 (1981).

[17] T.L. Dull, W.E. Frieze, D.W. Gidley, J.N. Sun, A.F. Yee, J. Phys. Chem. B 105, 4657 (2001).

[18] T. Goworek, K. Cieselski, B. Jasinska, J. Wawryszczuk, Chem. Phys. 230, 305 (1998).

[19] R. Zaleski, M. Sokół, Mater. Sci. Forum 666, 123 (2011).

[20] K. Ito, H. Nakanishi, Y. Ujihira, J. Phys. Chem. B 103, 4555 (1999).

[21] K. Sudarshan, D. Dutta, S.K. Sharma, A. Goswami, P.K. Pujari, J. Phys.: Cond. Matt. 19, 386204 (2007).

[22] K. Wada, T. Hyodo, J. Phys.: Conf. Ser. 443, 012003 (2013).

[23] K. Ito, Y. Yagi, S. Hirano, M. Miyayama, T.Kudo, A. Kishimoto, Y. Ujihira, J. Ceram. Soc. Jpn. 107, 123 (1999). 